\title{
Oralité et parole philosophante dans les notes de séminaire de Jacques Derrida
}

\section{Malika Temmar}

\section{(2) OpenEdition}

1 Journals

\section{Édition électronique}

URL : http://journals.openedition.org/genesis/1386

DOI : 10.4000/genesis.1386

ISSN : 2268-1590

Éditeur :

Presses universitaires de Paris Sorbonne (PUPS), Société internationale de génétique artistique littéraire et scientifique (SIGALES)

Édition imprimée

Date de publication : 17 novembre 2014

Pagination : 87-92

ISBN : 9782840509714

ISSN : 1167-5101

\section{Référence électronique}

Malika Temmar, "Oralité et parole philosophante dans les notes de séminaire de Jacques Derrida », Genesis [En ligne], 39 | 2014, mis en ligne le 15 décembre 2016, consulté le 01 mai 2019. URL : http:// journals.openedition.org/genesis/1386 ; DOI : 10.4000/genesis.1386 


\title{
Oralité et parole philosophante dans les notes de séminaire de Jacques Derrida
}

\author{
Malika Temmar
}

$\mathrm{S}$ i les « avant-dire » des séminaires donnés par Jacques Derrida entre 1969 et 1987 étaient dactylographiés, puis corrigés et annotés à la main, les textes préparatoires des séminaires suivants ont tous été directement rédigés sur un ordinateur, ajouts et corrections étant alors directement intégrés au document matrice, sans qu'il soit possible d'en retrouver la trace. Quand elle est disponible, la version publiée de ces enseignements restitue dès lors une image très fidèle non pas du cours tel qu'il a été professé mais du document que Derrida avait sous les yeux au moment de prendre la parole : un texte « oralisé » mais complètement rédigé. On ne trouve en effet pas ici de phrases nominales comme chez Merleau-Ponty, dont les avant-dire, simple feuille de route du séminaire, s'apparentent à une simple « prise de notes » à partir de laquelle le philosophe peut à sa guise improviser ou développer.

Destiné à être prononcé devant le public nombreux qui suit le séminaire, le texte de Derrida ne semble, en revanche, prévoir aucune place pour l'improvisation (même s'il est acquis que le philosophe sait, dès ce stade, qu'il aura des moments digressifs, etc.), mais il donne à lire, de façon paradoxale, une parole philosophique, dont la mise en scène est soigneusement prévue, en une sorte de mime d'un discours philosophique spontané. La part d'oralité inscrite dans l'avant-dire même du séminaire de Derrida, loin d'être marginale ou contingente, semble en effet faire partie intégrante de la démarche proprement philosophique. On s'efforcera alors de voir comment cette oralisation du texte participe non seulement de la construction d'un ethos philosophique, mais aussi d'une démarche de « déconstruction », qui invite à dépasser le rapport spontané au langage et remet en cause les habitudes de pensée.
Pour mettre ceci en évidence, il m'est apparu pertinent de prendre un appui privilégié sur la séance du 19 décembre 2001 de «La bête et le souverain », le dernier séminaire donné par Jacques Derrida à l'EHESS entre 2001 et 20031. Le texte de cette séance présente, d'une part, l'avantage d'avoir été publié par Derrida lui-même dans un cadre autre que celui du séminaire mais sans se départir de ses marques d'oralité 2 . Il est, d'autre part, particulièrement intéressant pour une réflexion sur les genres oraux et leurs avant-dire, parce que Derrida y thématise la notion même de « séminaire ». J'étudierai, dans un premier temps, la construction d'une scène énonciative spécifique, sur laquelle la parole philosophante doit pouvoir se déployer et qui assimile l'activité spéculative à un exercice de libre commentaire dans le cadre d'une démarche prototypiquement didactique et prototypiquement orale. Je m'attacherai, dans un second temps, à dégager la part d'oralité proprement « philosophique » de cet avant-dire, en me demandant comment les opérations de répétition et de reformulation, qui sont le propre de l'oral spontané mais que le document écrit peut prévoir, non seulement visent à mettre en scène le travail même de la pensée en exercice, mais aussi à mettre en évidence la nécessaire démarcation du concept, sa mise en danger, son « bougé ».

1. Cette étude porte sur les notes rédigées à l'ordinateur par Derrida telles qu'elles ont été publiées par Michel Lisse dans La Bête et le souverain, t. I (2001-2002), Paris, Galilée, 2008, p. 59-96. L'éditeur précise que le texte est donné « tel qu'il fut écrit par Jacques Derrida en vue de la parole, de la lecture à voix haute, donc avec certaines marques d'oralité anticipée et quelques tournures familières » (p. 11). Nous n'avons pas eu directement accès à l'original de ces notes.

2. «La bête et le souverain», dans Marie-Louise Mallet (dir.), La Démocratie à venir. Autour de Jacques Derrida, Paris, Galilée, 2004, p. 59-98. 


\section{Le séminaire comme genre}

Bien des notes de cours de philosophes sont désormais accessibles aux lecteurs. Leur publication est en effet fréquente, même, semble-t-il, lorsque le philosophe s'y est opposé de son vivant. Nous lisons ainsi des avantdire d'Henri Bergson, bien que celui-ci ait émis une interdiction catégorique le 8 février 1937, quatre ans avant sa mort :

Je déclare avoir publié tout ce que je voulais livrer au public. [...] J'interdis la publication de tout cours, de toute leçon, de toute conférence qu'on aurait pu prendre en note, ou dont j'aurais pris note moi-même. [...]. Je prie ma femme et ma fille de poursuivre devant les tribunaux quiconque passerait outre aux interdictions que je viens de formuler ${ }^{3}$.

Comme pour l'ensemble des avant-dire, les documents préparatoires des prestations orales des philosophes en situation de cours prennent des formes variées, qui vont de simples notes (séries nominales, phrases pas ou peu construites, forte discontinuité textuelle...) au discours rédigé avec soin. Les avant-dire pédagogiques de Merleau-Ponty relèvent plutôt du premier modèle, ceux de Bergson du second, comme on en jugera en comparant ces deux extraits :

Il y a un état de l'humanité, où nous sommes, et qui est 1) destructeur de la philosophie au sens ordinaire et classique (d'ailleurs cette caractérisation de notre temps n'est pas explication de crise de la philosophie : il y a projet de l'être (phase de Seinsgeschichte) à l'origine, que notre temps ne fait que dévoiler).

2) caractère philosophique de la littérature, de l'art etc. Ma thèse : cette décadence de la philosophie est inessentielle ; est celle d'une certaine manière de philosopher (selon substance, sujet-objet, causalité). La philosophie trouvera aide dans poésie, art, etc., dans un rapport beaucoup plus étroit avec elles, elle renaîtra et réinterprétera ainsi son propre passé de métaphysique - qui n'est pas passé4.

Le fait psychologique étant connu par la conscience, la méthode par excellence employée en psychologie devra être une méthode d'observation intérieure. La conscience, ou comme on dit aussi la réflexion interne, voilà l'instrument de connaissance auquel il faudra toujours recourir dans la recherche des faits et des lois psychologiques. On donne quelquefois à cette méthode le nom de méthode subjective. Nous verrons qu'on entend par subjectif en philosophie tout ce qui est intérieur au moi, tout ce dont nous prenons connaissance par la conscience seule. Le moi et le sujet sont deux termes synonymes 5 .

Bien que complètement rédigés, les avant-dire des séminaires de Derrida se distinguent de ceux de Bergson, en ce qu'ils prennent acte de leur devenir oral dans un cadre générique dont la spécificité est pleinement assumée. Cette spécificité, c'est celle d'une scène énonciative particulière, d'abord définie par le lieu lui-même : le grand amphithéâtre de l'EHESS, auquel l'avant-dire réfère par des repères déictiques comme « ici », et dont certains éléments sont déjà inclus dans le propos : «voilà ce qui nous reste sur la table de ce séminaire $^{6} »$. Le temps de référence de l'avant-dire est d'ailleurs déjà celui de la prise de parole à venir, lui aussi désigné par un embrayage (« aujourd'hui », « tout à l'heure », « maintenant »...) qui fait du moment de profération le point de repère des événements passés ou à venir ("souvenez-vous », « [...] qui devra nous occuper longtemps. Dès la prochaine fois », $B S$, p. 61). L'une des fonctions de cet embrayage temporel est d'assurer la cohérence du développement feuilletonné qui est le propre de la situation pédagogique mais aussi la continuité d'une démonstration philosophique qui pourrait pâtir de la discontinuité définitoire du séminaire :

À ce point, et pour conclure aujourd'hui en rappelant que nous n'avons pas cessé de suivre, à pas de loup, la trace du loup que nous retrouverons en janvier en lisant « Le loup et l'agneau » de la Fontaine après le 11 septembre, je me contenterai de reconnaître très vite $[\ldots](B S$, p. 91$)$.

3. Cité par Henri Gouhier, « Avant-propos » à Henri Bergson, Cours 1. Leçons de psychologie et de métaphysique, Paris, PUF, 1990, p. 5.

4. Maurice Merleau-Ponty, Notes de cours (1959-1961), éd. Stéphanie Ménasé, Paris, Gallimard, coll. « Bibliothèque de philosophie », 1996, p. 39.

5. Henri Bergson, Cours 1. Leçons de psychologie et de métaphysique, op. cit., p. 35.

6. La Bête est le souverain, op. cit., p. 91 (désormais $B S$ ). 
Mais il s'agit aussi de gérer la dimension interlocutive qui fonde le séminaire au moins comme fiction. Derrida note en effet des énoncés purement phatiques et n'appelant a priori pas de préparation au stade de l'avant-dire : «Voilà il est bien tard, bonne année » $(B S$, p. 91). C'est qu'il s'agit de prendre en considération, dès la phase de l'échange, les acteurs mêmes du séminaire. Unique orateur, Derrida ne cesse pourtant d'inclure le public à venir en le constituant comme co-énonciateur, c'està-dire comme co-constructeur de la démonstration philosophique. Aussi évite-t-il souvent le je au profit d'un nous de complicité, de connivence ou de cooptation (« l'une de nos questions »), mais renvoyant aussi au mythe fondateur de l'oralité philosophique, celui de la maïeutique.

Le séminaire philosophique doit en effet repenser le cadre communicationnel de la situation pédagogique : il ne saurait s'agir ici de dispenser un savoir. Aussi ne peutil toujours faire l'économie d'un retour sur lui-même :

En principe, dans la plus noble tradition de l'institution universitaire, un séminaire ne relève pas de la fable. Il n'appartient pas au genre de la fable. Il peut, certes, à l'occasion, se présenter comme un discours de savoir au sujet de cette loi du genre qu'on appelle fable ; il peut sans doute se donner pour un discours savant, historique, critique, théorique, philosophique, un discours de savoir sur la fable, au sujet du fabuleux en général.

Mais en principe, et suivant sa vocation statutaire, suivant sa loi et le contrat qu'il suppose, le discours enseignant ne doit pas être fabuleux. Il donne à savoir, il dispense le savoir, il faut savoir sans fable. Et il faut faire savoir sans fable. [...] L'une de nos questions pourrait alors s'annoncer ainsi, à l'intérieur d'un discours classique de séminaire, c'est-à-dire d'un discours théorique, philosophique, constatif, d'un discours de savoir, voire d'une réflexion de philosophie politique (BS, p. 67).

Il s'agit bien ici de négocier, de redéfinir voire de contractualiser avec le public la nature même de l'échange en cours. La parole philosophante ne peut être professorale sans cesser d'être philosophique (d'autant que ce séminaire tourne autour de la question du « faire savoir », au sens de démontrer). Il convient donc de gérer la tension entre deux exigences contradictoires, celle du discours d'autorité propre au professeur, celle du discours de co-construction du sens propre au philosophe. Cette tension, il convient de la gérer dès l'avant-dire en préparant avec soin l'ethos de la prise de parole à venir. Faut-il être professeur ? Faut-il prêter la voix au sens commun? Comment justifier sa propre place dans son propre discours? Quelles formes d'adresse utiliser?

À divers endroits du séminaire, Derrida utilise le je pour prendre en charge ses propos. La posture est alors parfois directive : «Je vous laisse suivre cela au début du livre chapitre XVIII »(BS, p. 77). Le je peut aussi atténuer la posture professorale en personnalisant ce qui est en fait proposé à l'accord de tous : «J'en retiens seulement ceci » $(B S$, p. 69) ; « je crois que c'est le meilleur mot» $(B S$, p. 65$)$; « Je livre maintenant à votre réflexion telle expression française » $(B S$, p. 60$) ;$ « Et ce que je voudrais souligner » $(B S$, p. 61$)$; « Je dirai dans un instant pourquoi je tiens à insister » $(B S$, p. 88). Le je est ici directement celui du philosophe en situation de discours. Ailleurs, l'embrayeur peut renvoyer au Derrida auteur, mais à titre parenthétique avant de revenir au je qui désigne directement l'orateur :

J'ai tenté ailleurs, dans des textes publiés ou non publiés, de montrer en détail en quoi cette distinction entre réaction et réponse reste dogmatique et donc problématique. Chez tous ceux que je viens de nommer. Je n'y reviens pas ici pour l'instant. Je me contente de remarquer que Hobbes est cartésien $[\ldots](B S$, p. 90).

Plus rarement, le je permet de référer à une expérience vécue : «ce que j’ai vu à la télévision alors que je me trouvais à New York, quelque quinze jours après le 11 septembre » $(B S$, p. 63). On trouve enfin quelques passages dans lesquels le je permet au philosophe d'entrer en polémique avec d'autres philosophes : «Je ne peux pas m'engager ici dans ce débat. Balibar pense que toute la doctrine de Bodin dément » $(B S$, p. 80$)$, « Je ne me hâterai pas de dire brutalement, comme je le pense, que tout cela est brutalement fait, qu'il est faux de dire que [...]» $(B S$, p. 83). La plupart des je de Derrida renvoient cependant à l'orateur en situation didactique immédiate, ce qui n'est pas le moindre des paradoxes d'un avant-dire, bien que le philosophe donne généralement préférence à des formes impersonnelles incluant le public qui alternent avec des nous de modestie. 
Autre paradoxe de l'avant-dire derridien, le public est inclus par des formes d'adresse qui sont donc prévues dès la phase préparatoire : " comme vous l'imaginez bien... », « comme vous le savez... », «si vous lisez au moins... », etc. Ces formes restent cependant, là encore, moins nombreuses que les nous ou les on omnipersonnels qui créent un effet de communauté : «La mise en œuvre de l'image, nous le savons bien » (BS, p. 63), «d'autre part, il $\mathrm{y}$ a cette imitation dont nous parlions tout à l'heure » $(B S$, p. 83), « nous n'avons pas cessé de suivre, à pas de loup, la trace du loup que nous retrouverons en janvier » $(B S$, p. 93), etc. Dans certains passages, en utilisant le nous, Derrida incarne un positionnement qu'il prête à son public par projection et cooptation : « La mise en œuvre de l'image, nous le savons bien », " On se demande quel aurait été le sens » (BS, p. 65). Ces passages se distinguent clairement des précédents, en ce que Derrida prête à ses interlocuteurs des positionnements interprétatifs. Dès l'avant-dire, Derrida prévoit donc sa posture : il évite de se poser en instance savante, et la parole philosophique est ici plutôt envisagée comme une pensée qui se réalise dans une sorte d'échange implicite, dans lequel le co-énonciateur participe au travail qui se réalise sous ses yeux dans une illusion de simultanéité qui est ici mimée. On constate en effet que le je embrayeur ne domine pas et qu'il est en fait souvent remplacé par de multiples formes d'adresse au public, ces formes étant elles-mêmes contrebalancées par des formes inclusives qui semblent co-construire le propos dans une illusion d'égalité.

\section{Reformuler pour formuler}

Le séminaire de Derrida présente, comme nous l'avons souligné, la particularité d'être extrêmement « pré-oralisé ». Cette caractéristique se constate également à la présence de pratiques de reformulation qui, si elles sont constitutives du discours philosophique, sont également rattachées à l'oral le plus ordinaire. À divers endroits, selon un phénomène réflexif, le discours de Derrida se retourne ainsi sur luimême, en se désignant comme procès de signification. Les formules, segments qui accompagnent ou introduisent à la méta-énonciation et à la glose, balisent ses propos.

Ces passages dans lesquels le philosophe porte son discours sur le discours lui-même sont particulièrement systématiques, au point qu'ils constituent des automatismes. Ces gloses apparaissent comme des moments d'arrêt dans lesquels s'effectue toute une opération interne de définition : une notion est préférée à une autre, et il y a en un sens et de façon implicite concurrence entre plusieurs termes possibles. Cette démarche procède du fait que toute signification de mot n'est pas univoque et que c'est là un terrain privilégié pour l'écriture philosophique : le philosophe ne peut construire ses thèses qu'à travers un travail sur et dans le langage :

Depuis Socrate, les philosophes ont cherché à maîtriser le langage qu'ils employaient et ont toujours plus ou moins lié la réflexion philosophique à une détermination du sens des mots et des concepts employés, certains et non des moindres allant même jusqu'à faire consister l'activité philosophique tout entière dans le procès de production de concepts7.

La fréquente reformulation présente donc un double bénéfice dans l'avant-dire derridien : d'une part, elle marque l'inscription dans le discours philosophique, en tant que le travail du concept est aussi un travail du lexique ; d'autre part, elle renforce l'oralité du propos, en ce que le retour correctif sur le dit est constant dans l'oral spontané. Rappelons que reformuler,

c'est formuler à nouveau et/ou formuler différemment, peut-on lire dans les dictionnaires; or si le préfixe re- a bien pour une de ses acceptions celle d'itération, formuler en revanche, engage toujours une situation d'énonciation singulière, forcément unique et distinctive. La reformulation s'inscrit donc dans un processus particulier qui dans le même temps qu'il pose un dit nouveau, re-dit un propos antérieur. Ainsi, la reformulation impose-t-elle dans son mieux-dit du déjà-dit $[\ldots]^{8}$.

Si la reformulation peut engager plusieurs formes d'opérations langagières (la répétition, la reformulation paraphrastique ou non paraphrastique, la reprise associée à une expansion, la glose, la variation linguistique...), il s'agit surtout pour Derrida de procéder à des substitutions (para)synonymiques, voire antonymiques ;

7. Jean-Gérard Rossi, La Philosophie analytique, Paris, PUF, coll. «Que sais-je ? », 1993 [1989], p. 4.

8. Marie-Claude Le Bot et al., La Reformulation : marqueurs linguistiques, stratégies énonciatives, Rennes, PUR, 2008, p. 11. 
les paraphrases ou gloses explicatives sont rares. Dans le passage suivant, le philosophe fait ainsi alterner plusieurs mots qui passent de synonymes à antonymes, comme c'est le cas de couple, d'abord simplement répété puis repris par duo (synonyme) puis par duel (antonyme), avant de laisser place à tête à tête puis face à face, etc. :

La bête et le souverain, la bête est le souverain, ainsi s'annoncerait d'abord notre couple, un couple, un duo, voire un duel, mais aussi une alliance, presqu'un hymen dont nous avons déjà commencé la semaine dernière à interroger le remuant tête à tête. Tête à tête ou face à face, hanté par de virtuelles différences sexuelles, entre, d'une part, la simple conjonction (e.t) qui les poserait, opposerait ou juxtaposerait comme deux espèces de vivants radicalement hétérogènes l'un à l'autre, l'un infrahumain, l'autre humain, voire sur-humain et, d'autre part, la copule (e.s.t) qui les accouplerait dans une sorte d'attraction ontologico-sexuelle, de fascination mutuelle, d'attachement communautaire, voire de ressemblance narcissique, l'un reconnaissant en l'autre une sorte de double, l'un devenant l'autre, étant l'autre (le « est » ayant alors valeur de processus, de devenir, de métamorphose identificatoire), la bête étant le souverain, le souverain étant la bête, l'un, l'autre se trouvant l'un et l'autre engagés, en vérité changés, voire échangés dans un devenir-bête du souverain ou dans un devenir-souverain de la bête, le passage de l'un à l'autre, l'analogie, la ressemblance, l'alliance, l'hymen tenant à ce qu'ils partagent tous deux cette très singulière position d'être hors-la-loi, au dessus ou à l'écart du droit, la bête ignorant le droit et le souverain ayant le droit de suspendre le droit, de se placer au dessus de la loi qu'il est, qu'il fait, qu'il institue, dont il décide souverainement. Le souverain n'est pas un ange mais, dirait-on, qui fait le souverain fait la bête. Le souverain se fait bête, se fait la bête, parfois au sens le plus trouble d'une zoophilie, voire d'une bestialité dont nous aurions à inventorier, détecter, voire interpréter les symptômes historiques. Voilà notre premier élan, voilà le nerf de notre analogie en et/est. Notre eh eh... (BS, p. 52).

Dans ce passage, le mot « couple », qui désigne la bête et le souverain, est repris de la façon suivante : « notre couple », « un couple », « un duo », « un duel », « une alliance », « un hymen », « tête à tête » et « face-à-face ». Le texte procède ensuite à des opérations de reprises dérivationnelles avec « poserait », qui devient « opposerait » et « juxtaposerait ». Ou encore : « engagés », « changés », « échangés », etc. Par la reformulation, Derrida fait donc varier un terme, en le répétant et aussi en le corrigeant au moyen de multiples opérations de substitutions et d'expansions nominales. Les reformulations sont souvent immédiates et contribuent à la continuité du discours. Certaines d'entre elles constituent des processus complexes de réélaboration qui demandent une coopération des allocutaires.

Le discours de Derrida procède ainsi par effet de structuration progressive au moyen de la reformulation. Bien que la reformulation soit aussi présente dans les écrits non oralisés de Derrida, l'oralité permet ce travail plus aisément que l'écrit ; constitutive de l'oral spontané, la reformulation tâtonnante y apparaît moins artificielle. L'intonation orale permet aussi d'insister sur le mot proposé comme reformulation. Il est cependant vrai que l'oral spontané privilégie l'épanorthose, qui est une simple reformulation corrective, alors que Derrida utilise aussi souvent la paradiastole, qui corrige par différenciation, en faisant éclater une notion tenue pour homogène en deux termes opposés. Ainsi dans l'exemple de couple et duo, l'opposition des deux termes est contextuelle et peu stabilisée, puisque les termes sont en général pris comme synonymes : le fait de reformuler l'un par l'autre permet à Derrida de faire apparaître ou de créer une différence qui est porteuse de sens dans la mesure où le second terme influe rétroactivement sur le premier et conduit à sa réinterprétation.

Le temps de la démonstration, nous avons ici suspendu une question importante : l'avant-dire de Derrida n'est-il pas en fait un avant-texte ? Destiné à être prononcé, le texte est également destiné à être publié, un jour, sans trop d'aménagements. C'est possible, mais c'est alors que l'avant-dire vaut texte : si l'on publie aujourd'hui tant de notes de cours de philosophes, et cela même quand ceux-ci ont manifesté par avance leur plus forte opposition, c'est que le séminaire nous paraît être le prototype de l'activité philosophante. Guidé par la parole magistrale, il garde la formalité et la rigueur de l'écrit, tout en conservant les allures spontanées de l'oral en ce qu'elles valent gage de sincérité. L'avant-dire derridien garde la trace de cet entredeux. Que le philosophe prévoit dès ce stade qu'il ira écrire un mot au tableau, voici qui trouble. Il n'importe : encore écrit, déjà oral, l'avant-dire du séminaire philosophique déplace la question même de la philosophie : il oblige à définir celle-ci comme un dispositif discursif autant que comme une démarche spéculative. 
Malika Temmar, maître de conférences en sciences du langage (Univ. d'Amiens/Céditec Paris XII), a une double formation en philosophie et littérature. Spécialisée dans l'analyse du discours philosophique, elle a publié Le Recours à la fiction dans le discours philosophique (Lambert-Lucas, 2013). Elle est l'auteure de plusieurs articles sur les formes expressives de la philosophie. Son approche met en lumière la façon dont les concepts philosophiques sont élaborés dans l'exercice d'une écriture. Sa réflexion porte, dans une perspective plus large, sur les outils et méthodes de l'analyse du discours. Elle a cherché à étudier, dans une perspective pluridisciplinaire, la façon dont l'analyse du discours peut être mobilisée dans des domaines variés, comme la critique littéraire médiatique, les blogs littéraires ou l'économie (lire : «La représentation stéréotypée de l'écrivain dans la critique littéraire médiatique », 2009 ; «Qui parle dans les blogs ?», 2012 ou Les Discours sur l'économie (co-dir.), PUF, 2014).

malikatem@yahoo.fr

\section{Oralité et parole philosophante dans les notes de séminaire de Jacques Derrida}

Ce texte analyse l' " avant-dire » d'un des séminaires donnés par Jacques Derrida, un texte préparatoire rédigé sur un ordinateur dont les ajouts et les corrections ont été directement intégrés au document matrice, sans qu'il soit possible d'en retrouver la trace. La version publiée de ces enseignements restitue une image très fidèle du document que Derrida avait sous les yeux au moment de prendre la parole : un texte « oralisé » mais complètement rédigé. Si le texte de Derrida ne semble prévoir aucune place pour l'improvisation, on y trouve une parole philosophique, dont la mise en scène est soigneusement prévue, en une sorte de mime d'un discours philosophique spontané. La part d'oralité inscrite dans l'avant-dire, loin d'être contingente, semble faire partie intégrante de la démarche proprement philosophique. On s'efforce ici de voir comment cette oralisation du texte participe non seulement de la construction d'un ethos philosophique, mais aussi d'une démarche de déconstruction.

This text analyses the "avant-dire" of one of Jacques Derrida's seminars, a preparatory text written on a computer, its additions and corrections having been directly integrated in the "matrix document", so that it is impossible to find any trace of them. The published version of these lessons reproduce a very faithful image of the document Derrida had before him when he spoke: an "oralised" text entirely written. If Derrida's text seems to have left no room for improvisation, we can find in it a philosophical spoken word, the staging of which was carefully planned, in a sort of mime of a spontaneous philosophical speech. The oral part inscribed in the "avant-dire", far from being contingent, seems to be an integral part of the strictly philosophical approach. We will try to see how this oralisation of the text plays a role not only in the construction of a philosophical ethos, but also in a deconstruction approach.

Dieser Artikel untersucht das „Avant-Dire“ eines der von Jacques Derrida gehaltenen Seminare, ein auf Computer geschriebener Vorbereitungstext, wo die Anmerkungen und Korrekturen direkt in das Originaldokument integriert wurden und es somit nicht möglich ist, deren Spuren wiederzufinden. Die veröffentlichte Version dieser Seminare gibt ein sehr wortgetreues Bild des Dokumentes wieder, das Derrida beim Sprechen vor Augen hatte: ein „oralisierter“ aber komplett überarbeiteter Text. Obschon der Text von Derrida keinerlei Platz für Improvisation vorzusehen scheint, findet man darin ein philosophisches Wort, dessen Inszenierung sorgfältig geplant ist, in einer Art Imitation eines spontanen philosophischen Diskurses. Der im Avant-Dire enthaltene mündliche Aspekt, in keiner Weise zufällig, scheint ein fester Bestandteil des eigentlichen philosophischen Vorgehens zu sein. Es soll hier untersucht werden, wie diese Vermündlichung des Textes bezeichnend ist nicht nur für die Konstruktion eines philosophischen Ethos, sondern auch für eine Art der Dekonstruktion
Este texto analiza el "pre-decir" de un seminario dictado por Jacques Derrida, un texto preparatorio redactado en la computadora, cuyos añadidos y correcciones han sido incorporados directamente en el documento, sin dejar ningún rastro identificable. La versión publicada de estos cursos restituye una imagen muy fiel del documento que Derrida tenía ante sus ojos en el momento de tomar la palabra: un texto "oralizado", pero totalmente redactado. Si el texto de Derrida, aparentemente, no da lugar en absoluto a la improvisación, pone sí de manifiesto una palabra filosófica, cuya puesta en escena está cuidadosamente prevista, en una suerte de mimo de un discurso filosófico espontáneo. La parte que corresponde a la oralidad, inscrita en el pre-decir, lejos de ser contingente, parece formar parte integrante del proceso propiamente filosófico. Intentaremos mostrar aquí cómo esta oralización participa no solamente en la construcción de un ethos filosófico, sino también de un proceso de deconstrucción.

O texto analisa o "ante-dito" de um seminário de Jacques Derrida - texto preparatório escrito em computador com adições e correções diretamente incorporadas no documento matriz, de impossível rastreamento. A versão publicada das lições dá uma imagem muito fiel do documento que Derrida lia no momento de usar da palavra: um texto "oralizado", mas completamente redigido. Se o texto de Derrida não reserva espaço para a improvisação, então ele é um discurso filosófico, de cuidada encenação, que mimetiza um discurso filosófico espontâneo. A parte de oralidade inscrita no ante-dito, longe de ser contingente, parece fazer parte integrante do processo propriamente filosófico. Procura-se aqui ver como esta oralização do texto contribui não só para a construção de um ethos filosófico, mas também de uma tentativa de desconstrução.

Questo testo analizza l'avandetto di uno dei seminari di Jacques Derrida, un testo preparatorio redatto al computer le cui aggiunte e correzioni sono state direttamente integrate nel documento principale, e per questo motivo è impossibile ritrovarne la traccia. La versione pubblicata di questi corsi restituisce un'immagine molto fedele del documento che Derrida aveva sotto gli occhi al momento di prendere la parola: un testo "oralizzato" ma completamente redatto. Anche se il testo di Derrida non sembra contemplare nessuno spazio per l'improvvisazione, vi si trova una parola filosofica la cui messa in scena è scrupolosamente preparata, in una sorta di mimica di un discorso filosofico spontaneo. La parte di oralità inscritta nell'avandetto, lungi dall'essere contingente, sembra essere parte integrante dell'approccio strettamente filosofico. Tenteremo qui di vedere in che modo questa "oralizzazione" del testo partecipa non solo della costruzione di un ethos filosofico ma anche di una pratica di decostruzione. 